\title{
Utilización de fascia lata alogénica para la herniorrafia perineal canina: comunicación de 7 casos clínicos
}

\author{
Use of allogenic fascia lata in perineal herniorrhaphy in dogs: \\ communication of 7 clinical cases
}

\author{
GG Semiglia $^{\text {a*}}$, DF Izquierdo ${ }^{\mathrm{a}}$, JH Zunino ${ }^{\mathrm{b}}$
}

\begin{abstract}
aUniversidad de la República Oriental del Uruguay, Departamento de Pequeños Animales, Facultad de Veterinaria, Universidad de la República Oriental del Uruguay, Montevideo, Uruguay.

${ }^{\text {b} B a n c o ~ d e ~ O ́ r g a n o s ~ y ~ T e j i d o s, ~ F a c u l t a d ~ d e ~ M e d i c i n a, ~ H o s p i t a l ~ d e ~ C l i ́ n i c a s, ~ M o n t e v i d e o, ~ U r u g u a y . ~}$
\end{abstract}

\begin{abstract}
SUMMARY
The aim of this study was to describe and evaluate the treatment of perineal herniorrhaphy in dogs using allogeneic fascia lata. For this purpose, fascia lata was obtained from dog weighing $15 \mathrm{~kg}$ or more, whose death cause did not involve infectious or oncologic conditions, nor collagen- disease or trauma, or surgical scars to the thighs. The fascia lata was preserved in $75 \%$ glycerin solution. Seven patients with unilateral perineal hernia were selected for this clinical trial. Using a perineal approach, the hernia was reduced, muscles repositioned, and reconstruction was achieved suturing the fascia lata with interrupted 0 Nylon monofilament suture. Evaluations were made at 7, 15, 30 and 60 days, to assess the following parameters: defecation, micturition, local inflammation, and pain. The allogeneic graft was not rejected, and the herniorrhaphy technique was successful in all cases. No post-surgical complications were observed at 60 days after, while defecation, micturition and regional anatomy returned to normality. Allogeneic processed fascia lata has herein demonstrated to be biocompatible and well tolerated, thus allowing to be considered a safe and useful option to perineal herniorrhaphy technique in dogs.
\end{abstract}

Palabras clave: herniorrafia, injerto, alogénico, canino.

Key words: herniorrhaphy, graft, allogeneic, dogs.

\section{INTRODUCCIÓN}

La hernia perineal (HP) ha sido reportada en varias especies, siendo la canina la más afectada (Hosggod y col 1995, Fossum y col 1999, Duval y col 2001). Esta patología resulta de la debilidad y separación de los músculos y la fascia que conforman el diafragma pélvico, permitiendo que el recto y contenido pélvicos y/o abdominales se desplacen hacia caudal (Hedlund 2004). El diafragma pélvico está compuesto por los músculos elevador del ano, coccígeo y la fascia perineal profunda y superficial (Hedlund 2004). Cualquier canino de raza pura o mestiza puede afectarse, pero hay ciertas razas predispuestas como el Terrier de Boston, Collie, Bóxer, Pekinés, Corgi galés, Kelpie, Caniche miniatura, Pastor alsaciano, Boyero de flandes, Antiguo pastor inglés, Dachshund y mestizos (Anderson y col 2001, Hedlund 2004). La HP afecta fundamentalmente a machos adultos a partir de los 5 años de edad, en su mayoría enteros, aunque pueden verse afectados también los castrados, y se han documentado unos pocos casos en hembras (Brown y

Aceptado: 06.10.2010.

* Juan Spikerman 2180, CP 11.700, Montevideo, Uruguay. gsemigli@adinet.com.uy
Udall 1964, Anderson y col 2001). Los contenidos de la HP son en orden de prevalencia la grasa retroperitoneal, líquido seroso, recto, próstata, vejiga urinaria e intestino delgado (Anderson y col 2001). Los signos clínicos incluyen tumefacción en región perineal unilateral o bilateral con predominio del lado derecho, tenesmo fecal y urinario, constipación, obstipación, disquecia y retención urinaria (Bellenger y Canfield 2003, Hedlund 2004).

La causa específica de la hernia perineal aún no ha sido establecida. Sin embargo, factores causales postulados incluyen: desequilibrio de hormonas gonadales (Hosggod y col 1995), esfuerzo contra el diafragma pélvico como resultado de prostatomegalia (Dorn y col 1985) y/o enfermedad rectal (Hosggod y col 1995), y variación anatómica de la musculatura del diafragma pélvico por atrofia muscular de origen neurogénico (Bellenger y Canfield 2003, Hedlund 2004).

El tratamiento de elección para la HP es el quirúrgico, existiendo varias técnicas para su resolución; las dos más comunes son la herniorrafia convencional (estándar) (Weaver y Omamegbe 1981) y la transposición del músculo obturador interno (Anderson y col 2001). Otras técnicas descritas son: la transposición de colgajos musculares tales como los músculos semitendinoso (Mann y Constantinescu 1998), glúteo superficial (Weaver y Omamegbe 1981) o la combinación de ambos; la utilización de flap miocutáneo del músculo gracilis (Bellenger y Canfield 2003). 
Diferentes técnicas y materiales tales como colágeno porcino (Frankland 1986), submucosa intestinal porcina (Stoll y col 2002), mallas de polipropileno (Matera y col 1981, Fossum y col 1999, Shoukry y col 1997) y fascia lata autógena (Bongartz y col 2005) se han utilizado en la plastia de hernias perineales en caninos.

Se han reportado varios usos de fascia lata en cirugía veterinaria, tales como en la reparación de ligamento cruzado craneal (Carvalho Buquera y col 2002), reparación de tendón calcáneo común (Shani y Shahar 2000), reparación de defectos uretrales (Gultekin y col 2005), reemplazo total de ligamento patelar (Gemmil y Carmichael 2003), estabilización de articulación coxofemoral (Seullner Brandão y col 2002) y en herniorrafia perineal (Bongartz y col 2005). En este último caso de forma autógena. Sin duda los materiales biológicos obtenidos de fuentes seguras y procesados adecuadamente aseguran su biocompatibilidad y buena tolerancia en injertos e implantes alogénicos.

El objetivo de este estudio fue determinar la biocompatibilidad de la fascia lata alogénica conservada en glicerina al $75 \%$, para ser utilizada en la herniorrafia perineal canina.

\section{MATERIAL Y MÉTODOS}

Para la extracción de fascia lata fueron elegidos animales mayores de $15 \mathrm{~kg}$, cuyo motivo de muerte no fue ninguna enfermedad infecciosa u oncológica, además de carecer de colagenopatías y presencia de traumatismos o abordajes quirúrgicos previos a nivel del muslo. Los cadáveres fueron obtenidos del Hospital del Departamento de Pequeños Animales de la Facultad de Veterinaria de la República Oriental del Uruguay, con previa autorización firmada de los propietarios.

La extracción de la fascia lata se realizó dentro de las primeras 6 horas de la muerte del animal, respetando medidas de asepsia quirúrgica que incluyeron rasurado del miembro posterior desde la cadera hasta la articulación tibio-tarsal y antisepsia con solución acuosa de povidona yodada al $1 \%$. Se procedió a la colocación de campos estériles, se identificaron los reperes óseos, hacia proximal el trocánter mayor del fémur y hacia distal la articulación fémoro-tibio-rotuliana. Se realizó incisión de piel y celular subcutánea, siguiendo una línea longitudinal que una a ambos puntos de referencia - proximal y distal- con una leve curvatura hacia craneal. Llegado al plano de la aponeurosis, se cambia la hoja de bisturí. Se extrajo el tejido adiposo que la cubre, quedando así expuesta la fascia lata en toda su extensión. Para facilitar el procesamiento es importante liberar en forma minuciosa el tejido adiposo de la fascia in situ. Luego se procedió a la identificación de la unión musculotendinosa, de los bordes craneal y caudal, incidiendo los mismos con bisturí, de proximal a distal. La fascia lata se libera del plano profundo hacia proximal y se secciona transversalmente a nivel de la unión musculotendinosa.
El material extraído se colocó sobre campos estériles, se procedió a cambiar guantes y por medio de rugina se retiraron restos de tejido adiposo. La remoción completa del tejido adiposo se obtuvo sumergiendo el tejido en solución de éter y alcohol etílico, en proporción 1:1 por un período de 24 hs a $4^{\circ} \mathrm{C}$. Posteriormente se lava con 1 litro de solución fisiológica en tres etapas, quedando sumergida en suero fisiológico durante $15 \mathrm{~min}$. Se tomaron muestras para estudio microbiológico con tapa rotulada, con el autogenerado del donante y fecha de extracción. Toda la técnica de colección y procesamiento de la fascia lata fue según lo reportado por Zunino y col 2001. Posteriormente la fascia fue colocada en frasco estéril con tapa, conteniendo glicerol al 75\% (en proporción 1:50) (Zunino y col 2001), que fue previamente esterilizado en autoclave modelo Scanlan Morris, Ohlo Chemical \& Surgical Equipment Co, Madison, Wisconsin, U.S.A, a $120^{\circ} \mathrm{C}$ y 1 atmósfera de presión durante 20 minutos. El tiempo de conservación máximo a una temperatura de $-4{ }^{\circ} \mathrm{C}$ hasta su utilización fue de 6 meses. Después de este tiempo el material es descartado.

Siete caninos machos con un rango de edades de 7 a 12 años, y rango de peso de 4 a $40 \mathrm{~kg}$, que ingresaron al Hospital de Pequeños Animales de la Facultad de Veterinaria de la República Oriental del Uruguay con motivo de consulta de HP unilateral, participaron del trabajo. Fueron evaluados clínicamente, para descartar otras patologías coexistentes (cuadro 1). El estado general de los pacientes fue analizado mediante estudios de Hemograma, Plaquetas, Función renal y Función hepática.

El protocolo anestésico utilizado fue basado en acetilpromazina ${ }^{1}$ a dosis de 0,05 miligramos por kilo ( $\mathrm{mg} / \mathrm{kg}$ ) vía intramuscular (IM) y clorhidrato de ketamina ${ }^{2}$ a dosis de $5 \mathrm{mg} / \mathrm{kg}$ vía IM como premedicación; la inducción fue realizada 20 minutos después con lidocaína ${ }^{3}$ al $2 \%$ a dosis de $2,2 \mathrm{mg} / \mathrm{kg}$ vía intravenosa (IV) lenta y tiopental ${ }^{4}$ al $2,5 \%$ a efecto por vía IV; para el mantenimiento fue utilizado isofluorano ${ }^{5}$ entre $1,5 \%$ a $3 \%$ con una mezcla de oxígeno del 1\%; la analgesia fue realizada junto a la premedicación y fue realizada con dipirona ${ }^{6}$ a dosis de $25 \mathrm{mg} / \mathrm{kg}$ IV y clorhidrato de rramadol $^{7}$ a dosis de $2 \mathrm{mg} / \mathrm{kg} \mathrm{IV}$; 30 minutos antes de comenzar el acto quirúrgico fue administrado bencilpenicilina procaínica y dihidroestreptomicina ${ }^{8}$ a una dosis de $11.000 \mathrm{UI} / \mathrm{kg}$ (unidades internacionales por kilo).

La región perineal fue tricotomizada y preparada de forma aséptica, y el paciente fue colocado en decúbito ventral con la cola llevada hacia craneal. Posterior a la

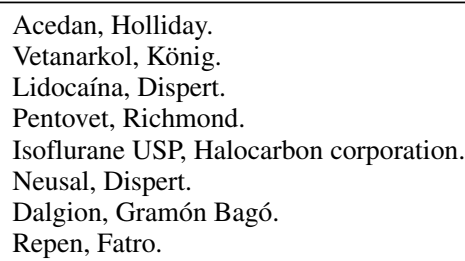


Cuadro 1. Reseña y datos patológicos de 7 caninos machos intervenidos quirúrgicamente para reparación de hernia perianal Clinical and pathological data of seven patients undergoing herniorrhaphy surgery

\begin{tabular}{lclclll} 
Animal & Edad (años) & \multicolumn{1}{c}{ Raza } & Peso (Kg) & Contenido herniario & Patología rectal & Lado afectado \\
\hline 1 & 10 & Cruza & 15 & GR, R & Saculación & Derecho \\
2 & 8 & Ovejero Alemán & 40 & GR, P & Ausente & Derecho \\
3 & 12 & Cruza & 12 & GR, R, P, V & Divertículo & Derecho \\
4 & 7 & Cruza & 4 & GR, R, P & Saculación & Derecho \\
5 & 10 & Cruza & 17 & GR, R & Divertículo & Derecho \\
6 & 10 & Cruza & 5 & GR, R & Divertículo & Derecho \\
7 & 7 & Ovejero Alemán & 35 & GR, R & Divertículo & Izquierdo \\
\hline
\end{tabular}

GR: grasa retroperitoneal

$\mathrm{R}$ : recto

P: próstata

V: vejiga

colocación de los campos quirúrgicos, se realizó incisión en piel sobre la hernia desde lateral hasta la base de la cola, casi por debajo de la masa herniaria; la incisión es curvada ligeramente hacia lateral en dirección dorsoventral. Una vez que fueron identificados los músculos comprometidos y liberados de sus adherencias, se procedió a la reducción de la hernia, para posteriormente suturar la fascia lata en el defecto. Para permitir una mejor manipulación de la fascia lata ésta fue sumergida en solución de suero fisiológico por 20 minutos para su hidratación, para posteriormente ser suturada a los músculos esfínter anal externo, coccígeo, obturador interno y ligamento sacrotuberal mediante puntos simples de vicryl $\mathrm{N}^{\circ} 0$ (figura 1). El tamaño de la fascia lata a suturar va a ser directamente proporcional al tamaño del defecto. Al momento de pasar los puntos por el músculo obturador interno y ligamento sacrotuberal se debe tener cuidado con los nervios perineal y pudendo, y la arteria y vena pudendas internas. Antes de realizar la síntesis rutinaria de subcutáneo y piel se realizó palpación rectal para descartar la posibilidad de presencias de sutura en la luz intestinal. En todos los casos la cirugía es acompañada de orquiectomía escrotal.

Los animales fueron medicados en el postoperatorio vía oral con amoxicilina más ácido clavulánico ${ }^{9}$ a dosis de $22 \mathrm{mg} / \mathrm{kg}$ cada 12 hs por 15 días, carprofeno ${ }^{10}$ a razón de $2,2 \mathrm{mg} / \mathrm{kg}$ cada $12 \mathrm{~h}$ por 3 días y dipirona ${ }^{11}$ a una dosis de $25 \mathrm{mg} / \mathrm{kg}$ durante 7 días. La alimentación realizada en el postoperatorio fue de ración comercial con alto contenido en fibras en un intervalo de tres horas.

Los controles postoperatorios se realizaron a los 7, 15, 30 y 60 días. La valoración clínica fue realizada

\footnotetext{
Clavamox, Pfizer.

10 Rimadyl, Pfizer.

11 Novemina, Laboratorio Lazar.
}

considerando los siguientes criterios usados por Bongartz y col 2005: grado de defecación $(0=$ defecación normal, 1 = leve dificultad, $2=$ moderada dificultad y $3=$ severa dificultad); grado de inflamación local $(0=$ no hay inflamación, 1 = leve, 2 = moderada, $3=$ severa), grado de dolor $(0=\sin$ dolor, $1=$ dolor leve, $2=$ dolor moderado, $3=$ dolor grave). Los parámetros fueron evaluados por el médico veterinario y por el propietario.

\section{RESULTADOS Y DISCUSIÓN}

Del total de animales sometidos a cirugía, cinco fueron caninos sin raza definida y dos de la raza Ovejero Alemán. Las patologías rectales encontradas fueron: saculación (2) divertículo (4) y un animal no presentó alteraciones rectales. Seis de los pacientes presentaron hernia unilateral derecha y el restante presentó hernia unilateral izquierda. El contenido herniario fue: grasa retroperitoneal, recto, próstata y vejiga variando su combinación en cada paciente (cuadro 1). La técnica quirúrgica en todos los casos tardó aproximadamente una hora y treinta minutos, y en el transcurso de la misma no se presentaron complicaciones. En la primera semana del postoperatorio se identificaron los mayores grados de inflamación (figura 2), dolor y dificultades en la defecación, que fueron disminuyendo en las semanas siguientes llegando a cero en todos los casos en el momento del alta (cuadro 2).

El paciente $\mathrm{N}^{\circ} 2$ en la primera semana presenta dehiscencia de tres puntos de la sutura en piel que fue tratada con lavados con solución fisiológica al $0,9 \%$ tres veces por día, lográndose la cicatrización a los 25 días del postoperatorio, luego de cuatro meses de haber recibido el alta médica regresa a consulta por hernia perineal contralateral al sitio de la cirugía. El paciente $\mathrm{N}^{\circ} 3$ regresa a los 3 meses de haber recibido el alta médica con una falla renal, que 

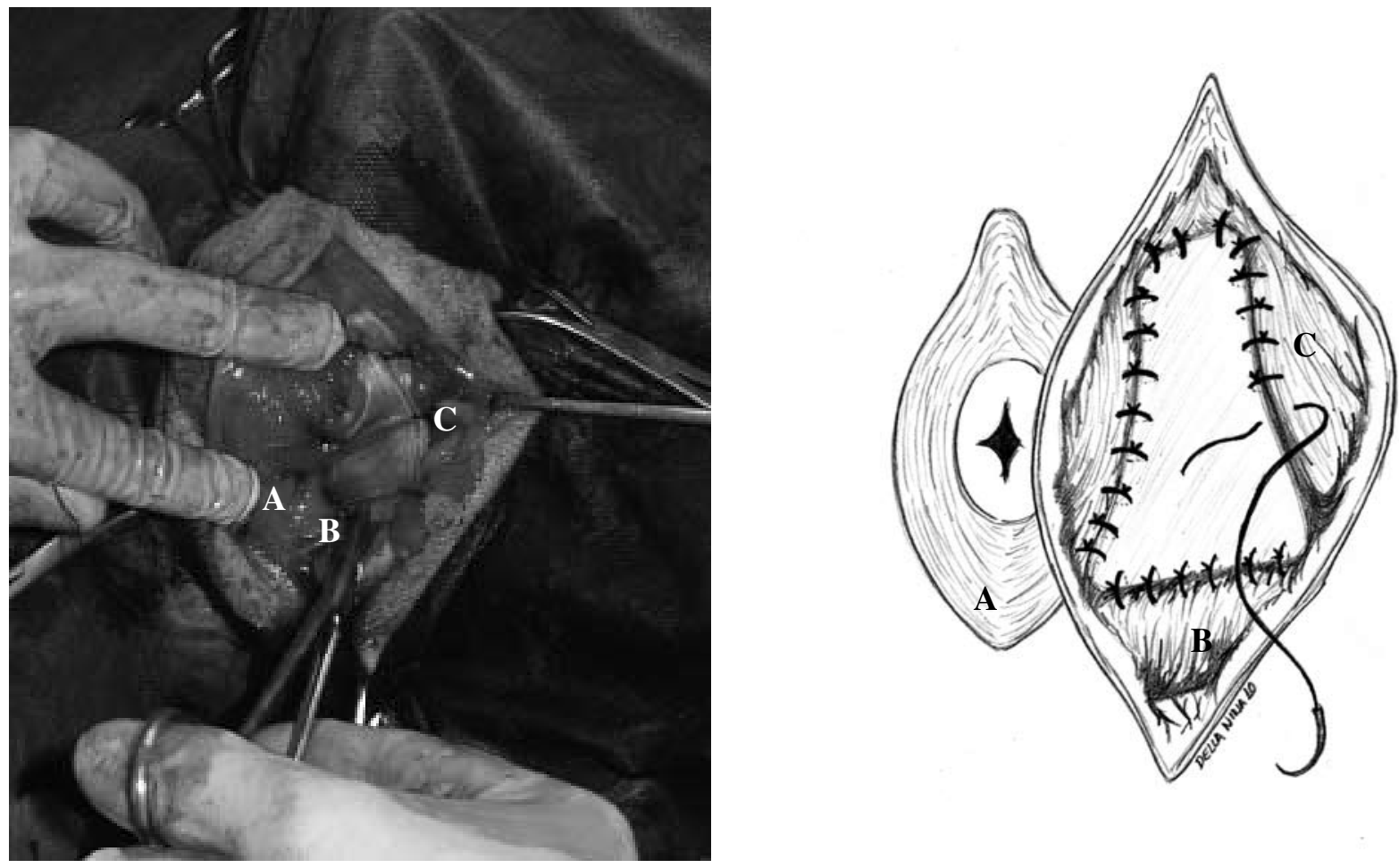

Figura 1. Sutura de la fascia lata a los músculos (A) esfínter anal externo, (B) obturador interno y (C) coccígeo, descritos en la técnica quirúrgica para herniorrafia perianal en caninos

Suture of the fascia lata to the muscles (A) external anal sphincter, (B) internal obturator, (C) coccygeal, described in the surgical techniques for perineal herniorrhaphy in dogs
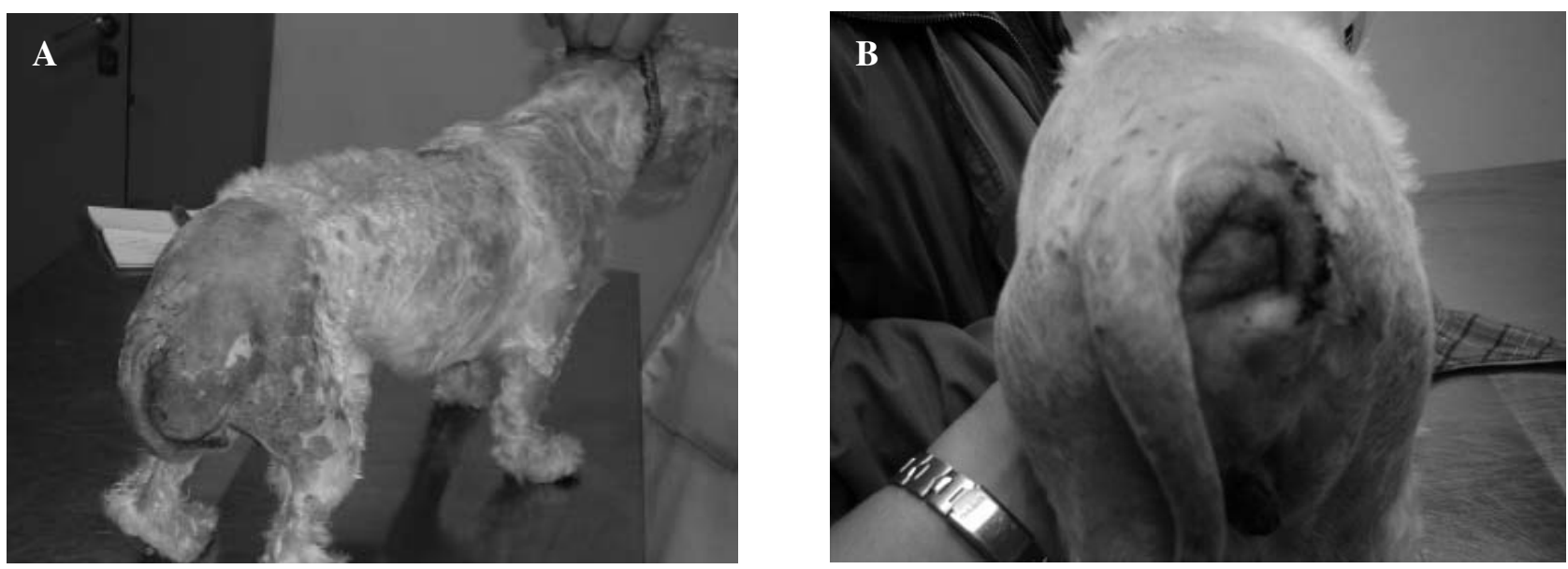

Figura 2. A - Preoperatorio, B - 15 días del postoperatorio de paciente canino hembra tratado quirúrgicamente por hernia perianal. A - Preoperative, B - 15 Posoperative days of a patient treated for herniorrhaphy.

no es posible compensar y fallece. A la fecha en el resto de los pacientes no se presentaron recidivas ni indicios de rechazo del injerto (fístulas y/o inflamación).

Tradicionalmente la técnica más empleada para la HP en caninos ha sido la herniorrafia tradicional en base a puntos simples de material absorbible o no absorbible. Varios autores como Bellenger (1980), Mattheison (1989),
Anderson y col (2001) y Hedlund (2002) reportan las complicaciones encontradas después de esta técnica como lesión del nervio pudendo, incontinencia fecal, infección en el sitio de la incisión, dehiscencia de la sutura, incontinencia urinaria y recidiva de la hernia. Estas complicaciones no fueron encontradas en nuestro trabajo con la técnica de fascia lata alogénica. La lesión del nervio pudendo se 
Cuadro 2 Evaluación postoperatoria (7, 15, 30 y 60 días) en los 7 caninos intervenidos quirúrgicamente para reparación de hernia perianal.

Postoperative evaluation $(7,15,30$ and 60 day) in 7 dogs after perineal herniorrhaphy.

\begin{tabular}{|c|c|c|c|c|c|c|c|c|c|c|c|c|c|c|}
\hline \multirow{3}{*}{$\begin{array}{c}\text { Animal } \\
1\end{array}$} & \multicolumn{12}{|c|}{ Signos clínicos } & \multirow{3}{*}{$\begin{array}{c}\text { Complicaciones } \\
\text { Ninguna }\end{array}$} & \multirow{3}{*}{$\begin{array}{c}\begin{array}{c}\text { Alta médica } \\
\text { postintervención } \\
\text { (días) }\end{array} \\
60\end{array}$} \\
\hline & \multicolumn{4}{|c|}{ Dolor } & \multicolumn{4}{|c|}{ Defecación } & \multicolumn{4}{|c|}{ Inflamación } & & \\
\hline & 2 & 0 & 0 & 0 & 1 & 1 & 1 & 0 & 3 & 1 & 0 & 0 & & \\
\hline 2 & 2 & 1 & 0 & 0 & 2 & 1 & 0 & 0 & 3 & 1 & 0 & 0 & Dehiscencia & 60 \\
\hline 3 & 2 & 0 & 0 & 0 & 2 & 1 & 1 & 0 & 3 & 1 & 0 & 0 & Ninguna & 30 \\
\hline 4 & 1 & 0 & 0 & 0 & 0 & 0 & 0 & 0 & 1 & 0 & 0 & 0 & Ninguna & 30 \\
\hline 5 & 0 & 0 & 0 & 0 & 1 & 0 & 0 & 0 & 1 & 0 & 0 & 0 & Ninguna & 60 \\
\hline 6 & 2 & 1 & 1 & 0 & 2 & 1 & 1 & 0 & 1 & 1 & 0 & 0 & Ninguna & 30 \\
\hline 7 & 2 & 2 & 1 & 0 & 2 & 1 & 0 & 0 & 2 & 2 & 1 & 0 & Ninguna & 60 \\
\hline
\end{tabular}

evita, porque no es necesario realizar suturas bajo tensión y abarcando gran cantidad de músculo, ya que el defecto creado va a ser cubierto por la fascia lata. La incontinencia fecal, urinaria y recidiva de la hernia no se presentó hasta el momento en ninguno de los 7 pacientes.

Las técnicas de colgajos musculares (músculos obturador interno, gluteosuperficial y semitendinoso) publicadas por Chambers y Rawling (1991) requieren experiencia por parte del cirujano para disminuir las complicaciones posquirúrgicas y el tiempo operatorio. Si bien no son reportadas grandes complicaciones con estas técnicas, el hecho de manipular los tejidos blandos de alrededor, genera una inflamación mayor, y el cirujano que realiza la técnica debe ser experimentado para disminuir los tiempos quirúrgicos. La simplicidad de la técnica mencionada en este trabajo permite que cirujanos con poca experiencia obtengan buenos resultados pre y posquirúrgicos con tiempos de cirugía relativamente cortos.

Materiales sintéticos como mallas de polipropileno reportado por Matera y col (1981), al ser un material extraño al organismo puede generar reacciones inflamatorias que complican la adecuada cicatrización y evolución. En nuestro caso el material utilizado si bien es externo al organismo del animal, es de la misma especie con un componente inmunológico bajo, por lo que no genera grandes reacciones inflamatorias como fue observado. Más recientemente se comenzó con la utilización de materiales biológicos tales como la submucosa intestinal porcina reportada por (Stoll y col 2002), colágeno dermal porcino (Frankland 1986) y fascia lata autógena (Bongartz y col 2005). Los materiales biológicos tienen la ventaja de no provocar grandes reacciones inflamatorias como los sintéticos. Con respecto a la mucosa intestinal porcina y el colágeno porcino, son injertos xenogénicos, los cuales tienen un mayor índice de rechazo por su mayor antigenicidad que los implantes alogénicos (Frankland 1986).

La fascia lata autógena implica un aumento de la morbilidad del sitio dador, así como aumenta el tiempo operatorio (Bongartz y col 2005), lo que no ocurre con la técnica de fascia lata alogénica.

Según los resultados obtenidos en 7 pacientes operados y evaluados en nuestro trabajo podemos concluir que la fascia lata alogénica conservada en glicerina al $75 \%$ resulta un buen injerto para la utilización en la herniorrafia perineal canina, disminuyendo tiempo y complicaciones quirúrgicas. Esta técnica no produce grandes reacciones inflamatorias manifiestas que indiquen un rechazo en el organismo receptor, permitiendo una buena evolución a mediano plazo, sin recidivas de la hernia.

Estudios posteriores serán necesarios con el propósito de aumentar el número de animales intervenidos, compararla con otras técnicas con objetivo de evaluar objetivamente sus ventajas y desventajas y realizar estudios histopatológicos.

\section{RESUMEN}

Este estudio describe y evalúa la técnica para la herniorrafia perineal utilizando fascia lata alogénica. Para esto se procedió a la extracción quirúrgica de fascia lata de caninos mayores de $15 \mathrm{~kg}$, que posteriormente fue conservada en glicerina al $75 \%$. Se seleccionaron siete pacientes caninos machos con hernia perineal unilateral. Los animales fueron sometidos a cirugía mediante un abordaje perineal, reduciendo la hernia, reposicionando los músculos y utilizando la fascia lata alogénica como material de reconstrucción del diafragma pélvico fijada a puntos simples con nylon monofilamento $\mathrm{N}^{\circ} 0$. Se realizaron controles a los $7,15,30$ y 60 días postcirugía, evaluando los siguientes parámetros: defecación, inflamación local y dolor. El injerto alogénico fue compatible en todos los casos tratados, lográndose una herniorrafia exitosa, sin complicaciones posoperatorias a los 60 días. En todos los casos la defecación, micción y anatomía regional volvieron a la normalidad. Este material demostró ser biocompatible y muy bien tolerado, permitiéndonos disponer de una opción segura para la técnica de herniorrafia perineal en el perro.

\section{AGRADECIMIENTOS}

A Marcos Ishimoto Della Nina, MV, por la realización del diseño gráfico. 


\section{REFERENCIAS}

Anderson MA, GM Constantinescu, FA Mann. 2001. Reparación de hernia perineal en caninos. En: Bojrab MJ. Técnicas actuales en cirugía de pequeños animales. $4^{\mathrm{a}}$ ed. Inter-Médica, Buenos Aires, Argentina, Pp 510-518.

Bellenger CR. 1980. Perineal Hernia in dogs. Aust Vet 56, 434-438.

Bellenger CR, R Canfield. 2003. Perineal hernia. In: Slatter D (ed). Textbook of small animal surgery. $3^{\text {rd }}$ ed. Saunders, Philadelphia, USA, Pp 487-498.

Bongartz A, F Carofiglio, M Balligand, M Heimann, A Hamaide. 2005. Use of autogenous fascia lata graft for perineal herniorraphy in dogs. Vet Surg 34, 405-413.

Brown DR, ND Udall. 1964. An unusual inguinal hernia in a bitch. Vet Rec 76, 1244-1249.

Carvalho-Buquera LE, JC Canola, JG Padilha Filho, J Maziero-Furlani, IC Talieri, AL Selmi. 2002. Radiografia e macroscopia do joelho após estabilização extra-artiuclar utilizando fáscia lata, fio de poliéster trançado ou fio de poliamida para correção da ruptura do ligamento cruzado cranial em cães. Ciencia Rural, Santa María 32, 73-78.

Chambers JN, CA Rawlings. 1991. Applications of a semitendinosus muscle flap in two dogs. J Am Vet Med Assoc 199, 84-86.

Dorn AS, RE Cartee, DC Richardson. 1982. A preliminary comparision of perineal hernia in the dog and man. J Am Anim Hosp Assoc 18, 624-632.

Duval JM, MA Anderson, GM Constantinescu. 2001. Hernia perineal en felinos. En: Bojrab MJ (ed). Técnicas actuales en cirugía de pequeños animales. $4^{\mathrm{a}} \mathrm{ed}$. Inter-Médica, Buenos Aires, Argentina, Pp 523-525.

Fossum TW, CS Hedlund, DA Hulse, AL Johnson, HB Seim III, MD Willard, GL Carroll. 1997. Cirurgia do períneo, do reto e do ânus. In: Fossum TW. Cirurgia de pequenos animais. Editorial Roca, São Paulo, Brasil, Pp 371-385.

Frankland AL. 1986. Use of porcine dermal collagen in the repair of perineal hernia in dogs -a preliminary report. Vet Rec 119, 13-14.

Gemmill TJ, S Carmichael. 2003. Complete patellar ligament replacement using a fascia lata autograft in a dog. J of Small Anim Pract 44, 456-459.
Gultekin A, C Mete, S Mahmut, O Isa. 2005. Repair of urethral defects using fascia lata autografts in dogs. Vet Surg 34, 514-518.

Hedlund CS. 2002. Perineal hernia. In: Fossum TW (ed). Small animal surgery. $2^{\text {nd }}$ ed. Mosby, St. Louis, USA, Pp 433-437.

Hedlund CS. 2004. Hernia perineal: Diagnóstico y tratamiento. Waltham Focus $14,5-11$.

Hosgood G, CS Hedlund, RD Pechman, PW Dean. 1995. Perineal herniorrhaphy: periooperative data from $100 \mathrm{dogs}$. J Am Anim Hosp Assoc 31, 331-342.

Mann FA, GM Constantinescu. 1998. Técnicas de salvataje para la herniorrafia perineal defectuosa. En: Bojrab MJ (ed). Técnicas actuales en cirugía de pequeños animales. $4^{\mathrm{a}}$ ed. Inter-Médica, Buenos Aires, Argentina, Pp 518-523.

Matera A, PS De Moraes Barros, AJ Stopiglia, RE Randi. 1981. Hérnia perineal no cão tratamento cirúrgico mediante utilização de malha de polipropileno. Rev Fac Med Vet Zootec 18, 37-41.

Mattheison DT. 1989. Diagnosis and management of complications occurring after perineal herniorraphy in dogs. Princeton Junction. Comp Cont Educ Pract 11, 797-822.

Seullner Brandao CV, P Iamaguti, LM Alvarez De Figuereido. 2002. Substituição do ligamento da cabeça do fêmur com auto-enxerto de fáscia lata na luxação coxofemoral em cães. Ciência rural, Santa María 32, 275-280.

Shani J, R Shahar. 2000. Repair of chronic complete traumatic rupture of the common calcaneal tendon in a dog, using a fascia lata graft. Vet Comp Ortho Traum 13, 104-108.

Shoukry M, M El-Keiey, M Hamouda, S Gadallah. 1997. Commercial polyester fabric repair of abdominal hernias and defects. Vet Rec 140, 606-607.

Stoll MR, JL Cook, ER Pope, WL Carson, JM Kreeger. 2002. The use of porcine small intestinal submucosa as a biomaterial for perineal herniorrhaphy in the dog. Vet Surg 31, 979-390.

Weaver AD, JO Omamegbe. 1981. Surgical treatment of perineal hernia in the dog. J Small Anim Pract 22, 749-758.

Zunino JH, MC Saldías, O Wodowóz. 2001. Técnicas de selección, extracción procesamiento y conservación de Tejidos. Publicación de Circulación Interna, Bco. de Tejidos. Banco Nacional de Órganos y Tejidos, Hospital de Clínicas, Universidad de la República, Montevideo, Uruguay. 